\title{
Biomechanically-Constrained 4D Estimation of Myocardial Motion
}

\author{
Hari Sundar, Christos Davatzikos, and George Biros \\ Section for Biomedical Image Analysis, Department of Radiology, \\ University of Pennsylvania
}

\begin{abstract}
We propose a method for the analysis of cardiac images with the goal of reconstructing the motion of the ventricular walls. The main feature of our method is that the inversion parameter field is the active contraction of the myocardial fibers. This is accomplished with a biophysically-constrained, four-dimensional (space plus time) formulation that aims to complement information that can be gathered from the images by a priori knowledge of cardiac mechanics. Our main hypothesis is that by incorporating biophysical information, we can generate more informative priors and thus, more accurate predictions of the ventricular wall motion. In this paper, we outline the formulation, discuss the computational methodology for solving the inverse motion estimation, and present preliminary validation using synthetic and tagged MR images. The overall method uses patient-specific imaging and fiber information to reconstruct the motion. In these preliminary tests, we verify the implementation and conduct a parametric study to test the sensitivity of the model to material properties perturbations, model errors, and incomplete and noisy observations.
\end{abstract}

\section{Introduction}

Medical Imaging can help in the diagnosis of cardiac masses, cardiomyopathy, myocardial infarction, and valvular disease. Advances in medical imaging methods have enabled us to acquire high-resolution 4D images of the heart that capture the structural and functional characteristics of individual hearts. Our long term goal is the integration of the proposed framework with cine magnetic resonance imaging (cine-MRI), which is emerging as the method of choice for diagnosing a variety of cardiovascular disorders 112. However, our framework can be used with any cardiac modality that gives spatio-temporal information, for example tagged cine-MRI, stimulated echo, CT. Computational challenges limit analysis to $3 \mathrm{D}(2 \mathrm{D} \times$ time $)$ motion estimation, where in fact $4 \mathrm{D}$ analysis would be preferable 34. Segmentation of the ventricles and the myocardium is the first step toward quantitative functional analysis of cine-MRI data. However, segmentation is time consuming, thereby limiting clinical throughput [5]. Moreover, sometimes accuracy is limited by long-axis motion, and inter and intra-observer variability.

Related Work. To address these problems in motion reconstruction, one of the main thrusts in recent research has been 4D motion estimation using 
biomechanical models. There is significant work on the integration of imaging with cardiovascular mechanics. In [6], a piecewise linear composite biomechanical model was used to determine active forces and the strains in the heart based on tagged MRI information. In [7] MR images were combined with biomechanical models for cardiac motion estimation. Interactive segmentation was combined with a Bayesian estimation framework that was regularized by an anisotropic, passive, linearly elastic, myocardium model. The authors recognized the importance of neglecting active contraction of the left ventricle. In [8], the need for realistic simulations and the need for inversion and data assimilation was outlined. In 9, Kalman filters were used to recover the initial state of the heart and spatial abnormalities. That method however, is difficult to generalize to nonlinear inversion with time-dependent inversion parameters.

Overview of the Method. Given 4D imaging data of cardiac motion the main steps in our framework are the following: (1) segment the end-diastolic frame to myocardium, blood pool, and surrounding tissue; (2) register the segmented frame with a cardiac atlas in order to assign material properties and fiber-orientation information [10]; and (3) solve an inverse problem for forces along the fibers in the myocardium by minimizing an image-similarity functional, which is constrained by the biomechanical model of the heart. In this way, we explicitly couple raw image information with cardiac mechanics.

Contributions. We propose a biomechanically-constrained motion estimation algorithm that has the potential to address intra-individual motion estimation problems. We discuss formulation, numerical implementation, and we present preliminary verification tests that confirm the potential of the method. The novelty of our approach is in the formulation and the algorithms (solvers and parallel implementation). The main features of our scheme are (1) a patient-specific image-based inversion formulation for the active forces; (2) a octree-based, adaptive finiteelement forward solver that incorporates anatomically-accurate fiber information; (3) an adjoint/Hessian-based inversion algorithm; and (4) a 4D coupled inversion for all images. This work builds on our previous work on parallel octree-based methods [1].

\section{Formulation of the Inverse Problem}

The basic premise of our formulation is the following: The heart motion is induced by the active forces in the myocardium. If we knew the exact biomechanical model for the myocardial tissue (constitutive law, geometry, fiber orientations, material properties for the heart and surrounding tissues, endocardial tractions due to blood flow) and the active stretching time-space profile, then we could solve a system of partial differential equations (PDEs) that describe the motion of the myocardial tissue for the displacements given the fiber forces; we refer to this system of PDEs (typically nonlinear elasticity) as the "forward problem". Similarly, if we knew the displacements at certain locations in the myocardium, we could solve the so-called "inverse problem" to reconstruct active forces so that 
the motion due to the reconstructed forces matches the observed one. More generally, we have imaging data, typically cine-MRI, but not the displacements. We can still invert for the displacements; by solving a biomechanically-constrained image registration problem. In this context, an abstract formulation of the myocardium motion estimation problem is given by

$$
\min _{u, s} \mathcal{J}\left(I_{t}, I_{0}, u\right) \quad \text { subject to } \mathcal{C}(u, s)=0 .
$$

Here, $I_{t}:=I_{t}(x, t)$ is the cine-MR image sequence with $x, t$ denoting the spacetime coordinates, $I_{0}:=I(x, 0)$ is the end-diastolic frame, $u:=u(x, t)$ is the displacement (motion), $s=s(x, t)$ is the active fiber contraction, and $\mathcal{C}$ is the forward problem operator. Also, $\mathcal{J}$ is an image similarity measure functional. This is a classical PDE-constrained inverse problem. Notice that there is no need for elastic, fluid, or any kind of regularization for $u$. It is constrained through the biomechanical model $\mathcal{C} 1$

Objective Function. (Operator $\mathcal{J}$ ). Different image similarity metrics can be used depending on the modality, like sum of squared differences and mutual information [12]. In this paper for simplicity, we consider point correspondences. We compute point-correspondences for all time frames, i.e., $d_{j}(t):=u\left(x_{j}, t\right)_{i=1}^{M}$ at $M$ points. Then, the objective function is given by

$$
\mathcal{J}:=\int_{0}^{1}(Q u-d)^{2} d t:=\int_{0}^{1} \sum_{i=1}^{M}\left(u\left(x_{j}, t\right)-d_{j}(t)\right)^{2} d t,
$$

where $Q$ is the so called spatial observation operator.

Forward Problem. (Operator $\mathcal{C}$ ). We make several approximations in our biophysical model. We assume a linear isotropic inhomogeneous elastic material for the myocardium; we ignore the geometric nonlinearities in both material response and active forces; we model the blood pool as an incompressible material with very small stiffness and large dissipation. We recognize that these are very strong assumptions but the model is meant to be driven by image data and assist in the motion reconstruction. More complex models, in particular nonlinear elasticity, will be incorporated in the future, if necessary. In addition to the constitutive assumptions, we assume a model for the active forces: given the fiber contractility $s$ as a function of space and time, we define the active stretch tensor $U=\mathbf{I}+s n \otimes n$, whose divergence results in a distributed active force of the form $\operatorname{div}(s n \otimes n)$; here $\mathbf{I}$ is the 3D identity matrix. Taken together, these assumptions result in the following form for $\mathcal{C}$ :

$$
M \ddot{u}(t)+C \dot{u}(t)+K u(t)+A s(t)=0 \quad t \in(0,1) .
$$

Using a Ritz-Galerkin formulation, with $\phi$ and $\psi$ basis functions for $u$ and $s$ respectively, the expressions for $M, K$ and $A(n)$ are given by $M_{i j}=\int \mathrm{I}\left(\phi_{i} \phi_{j}\right)$,

${ }^{1}$ However, one can show that the problem is ill-posed on $s$. Here we regularize by discretization of $s$. 
$K=\int(\lambda+\mu) \nabla \phi_{i} \otimes \nabla \phi_{j}+\mu \mathrm{I}\left(\nabla \phi_{i} \cdot \nabla \phi_{j}\right), A_{i j}=\int(n \otimes n) \nabla \phi_{i} \psi_{j}$, and $C=$ $\alpha M+\beta K$, with $\alpha$ and $\beta$ viscous-damping parameters. Here, $\otimes$ is the outer vector product, and $\lambda$ and $\mu$ are the Lamé constants. Equation (3) is derived by the Navier linear elastodynamics equation [13. The domain of spatial integration (for $M, K$, and $A$ ) is the unit cube, corresponding to the image domain. In our formulation, we solve for the motion of all the tissue in the MR images. At the outer boundaries of the cube we impose homogeneous Neumann boundary conditions(zero traction). Also, we assume zero displacements and velocities as initial conditions.

Inverse Problem. The inverse problem is stated by (1) where $\mathcal{J}$ is given by (2) and $\mathcal{C}$ is given by (3). By introducing Lagrange multipliers $p$, the first-order optimality conditions for (1) can be written as:

$$
\begin{array}{rr}
M \ddot{u}(t)+C \dot{u}(t)+K u(t)+A s(t)=0, \quad \dot{u}(0)=u(0)=0, \\
M \ddot{p}(t)-C \dot{p}(t)+K p(t)+Q^{T}(Q u-d)=0, \quad \dot{p}(1)=p(1)=0, \\
A^{T} p(t)=0 .
\end{array}
$$

Equation (4) consists of a system of partial-differential equations for $u$ (cardiac motion), $p$ (adjoints), and $s$ (active fiber contraction). It is a $4 \mathrm{D}$ boundary value problem since we have conditions prescribed at both $t=0$ and $t=1$.

Discretization and Solution Algorithms. We discretize the forward and adjoint problems in space using a Ritz-Galerkin formulation. We have developed a parallel data-structure and meshing scheme, discussed in 11. The basis functions are trilinear, piecewise continuous polynomials. In time, we discretize using a Newmark scheme. The overall method is second-order accurate in space and time. The implicit steps in the Newmark scheme are performed using the method of Conjugate Gradients (CG) combined with a domain-decomposition preconditioner in which the local preconditioners are incomplete factorizations. The solver and the preconditioner are part of the PETSc package [14.

For these particular choices of objective function and forward problem the inverse problem (4) is linear in $p, u$, and $s$. We use a reduced space approach in which we employ a matrix-free CG algorithm for the Schur-complement of $s$; also called the (reduced) Hessian operator. Each matrix-vector multiplication with the Hessian requires one forward and one adjoint cardiac cycle simulation. One can show that the Hessian is ill-conditioned and the overall cost of the method can be quite high. Although, there are ways to accelerate the calculations, here we report results from a simple CG method. To reduce the computational cost for the calculations in the present paper, we used a reduced-order model for $s$ in which $\psi$ is a product of B-splines in time and radial functions in space (Gaussians). The activation $s$ is parmeterized only when we solve the inverse problem. For the forward problem, we use the underlying finite element basis. 


\section{Results}

We first describe the set of experiments performed to validate the motion estimation framework using synthetic datasets. This is followed by results using MR tagged images.

Tests on Synthetic Datasets for Verification. In order to assess the parametrized model of the forces, we use an ellipsoidal model of the left ventricle. The fiber orientations are generated by varying the elevation angle between the fiber and the short axis plane between $+60^{\circ}$ and $-60^{\circ}$ from the endocardium to the epicardium [15. For this model we selected a Poisson's ratio $\nu=0.45$ and a Young's modulus of $10 \mathrm{kPa}$ for the myocardial tissue and $1 \mathrm{kPa}$ for the surrounding tissue and ventricular cavity 2 . Raleigh damping $(C=\alpha M+\beta K)$ was used with parameters $\alpha=0$ and $\beta=7.5 \times 10^{-4}$. In order to drive the forward model, we generated forces by propagating a synthetic activation wave from the apex to the base of the ventricles. The number of time steps were set to be 50 . The motion field obtained by solving the forward problem was used to drive the inverse estimation. The relative residual tolerance for the forward solver (CG) was set to $10^{-8}$ and to $10^{-4}$ for the inverse solver.

Table 1. Error in recovery of activation for increasing number of radial basis functions. By changing the inversion solver accuracy, we can accelerate the calculation without compromising accuracy (e.g., the $4^{3}$ calculation).

\begin{tabular}{|c|c|c|}
\hline Basis Size & Relative Error (\%) & Time \\
\hline $2^{3}$ & 13.1 & $36 \mathrm{mins}$ \\
$4^{3}$ & 5.67 & $\approx 5 \mathrm{hrs}$ \\
$4^{3}$ & 11.2 & $108 \mathrm{mins}$ \\
$8^{3}$ & 9.66 & $141 \mathrm{mins}$ \\
\hline
\end{tabular}

Table 2. Error in the recovery of activation with partial observations of the displacements. Errors are reported on the ellipsoidal model for a grid size of 32 with $4^{3}$ basis functions.

\begin{tabular}{|c|c|}
\hline Observations & Relative Error (\%) \\
\hline Full & $5.36 \times 10^{-2}$ \\
$1 / 8$ & $6.21 \times 10^{-2}$ \\
$1 / 64$ & $8.51 \times 10^{-2}$ \\
\hline
\end{tabular}

We calculated the error in the estimation of the activations for different degrees of parametrization using the radial basis. In all cases, the time dependence of the force was parametrized by five B-splines. The relative error in the estimation of the activation for a $64^{3}$ grid was computed for spatial parametrizations of $2^{3}, 4^{3}$ and $8^{3}$ and is tabulated in Table 1. In addition, we investigated the error in the estimation when only partial observations are available. We compared estimations based on full and sparse observations with $12 \%$ and $6 \%$ samples against the analytical solutions. These results are tabulated in Table 2 In order to assess the sensitivity of the motion estimation framework, we estimated the motion for the synthetic model of the heart at a grid size of 64 with a radial basis parametrization of $4^{3}$ by adding noise to the system. We added a $5 \%$ random error on the estimates of the fiber orientation and to the material properties of

${ }^{2}$ We treat the blood pool as an incompressible solid with low shear resistance. 
the myocardium. In addition, we added a $1 \%$ noise to the true displacements. The system converged and the relative error, in the $L^{2}$ norm, increased from $5.67 \times 10^{-2}$ to $9.43 \times 10^{-2}$. Overall, the solution is not sensitive to the errors in the material properties (due to the well-posedness of the forward problem) and on the noise (due to the presence of model regularization).

Validation using Tagged MR Images. We acquired tagged MR sequences for 5 healthy volunteers, on a Siemens Sonata $1.5 \mathrm{~T}^{\mathrm{TM}}$ scanner, in order to validate our motion estimation algorithm. Three short axis and a single long axis grid tagged, segmented k-space breath-hold cine TurboFLASH images with image dimensions of $156 \times 192$ pixels and with pixel size of $1.14 \times 1.14 \mathrm{~mm}^{2}$ were acquired. The slice thickness was $8 \mathrm{~mm}$. The displacements (observations) at the tag intersection points within the myocardium were computed manually. An average of 70-tag intersection points were selected over the left and right ventricles on each plane resulting in around 300 observations in space. Three independent observers processed all five datasets to get three sets of observations. To account for observer variability, we treated the mean location of the landmark as the ground truth observations.

The fiber orientations required for the biomechanical model were obtained using using ex-vivo DTI data and a tensor mapping method [10]. The end-diastolic MR image was segmented manually to assign material properties to the myocardial tissue, the blood and the surrounding tissue.

In three separate experiments, we used $70 \%, 50 \%$, and $20 \%$ of the ground truth observations (selected per slice) as the data for the inversion. The observations that are not used during the inversion are the control observations and are used for assessing the goodness of the inversion. We used the B-spline and the radial bases to reduce the parameter space. We used a total of $4^{3}$ spatial parameters, each with 5 B-spline temporal parameters, giving us a total of 320 parameters. We used a grid size of $64^{3}$ for all inversions.

After inversion, one additional forward solve was performed using the dense estimates of the fiber contractions to obtain dense estimates of myocardial displacements. These displacements were compared with the ground truth displacements at the tag-intersection points. The relative error (as a percentage) and the absolute error in millimeters for all observations and restricted to only the control observations are shown in Table 3. The RMS errors over time for different levels of partial observations are plotted in Figures 1a, 1c.

An alternate way of interpreting the results is to analyze the left-ventricular volume, obtained using manual segmentation and by the using the motion estimates, as seen for one particular subject in Figure 1d. The volume estimates using $70 \%$ and $50 \%$ observations are in general agreement with those obtained using manual segmentation. The volume estimated using $20 \%$ observations does not match very well with the manual segmentation, although the general trend is captured even when using only $20 \%$ observations. 
Table 3. Results from tagged data using a $64^{3}$ forward mesh size and an inverse parametrization of $4^{3}$ spatial parameters and 5 temporal B-spline parameters. The percentage specifies the percentage of total observations that were used to drive the inverse optimization. The control observations are the remaining observations that were not used during the inverse optimizations and are used for validation purposes.

\begin{tabular}{|c|c|c|c|c|}
\hline & $\begin{array}{c}\text { Error (\%) } \\
\text { All obs. }\end{array}$ & $\begin{array}{c}\text { Error (\%) } \\
\text { Control obs. }\end{array}$ & $\begin{array}{c}\text { Error }(\mathrm{mm}) \\
\text { All obs. }\end{array}$ & $\begin{array}{c}\text { Error }(\mathrm{mm}) \\
\text { Control Obs. }\end{array}$ \\
\hline Observers & 9.21 & 8.41 & 1.39 & 1.26 \\
Algorithm 70\% & 12.46 & 14.22 & 1.88 & 2.13 \\
Algorithm 50\% & 16.37 & 21.02 & 2.47 & 3.15 \\
Algorithm 20\% & 41.61 & 51.19 & 6.28 & 7.67 \\
\hline
\end{tabular}

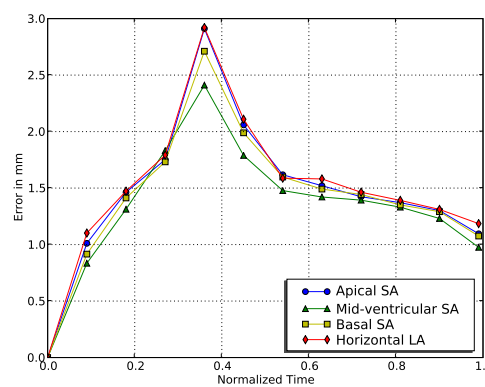

(a) Estimate with $70 \%$

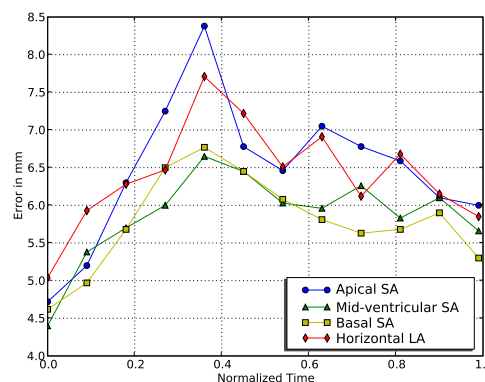

(c) Estimate with $20 \%$

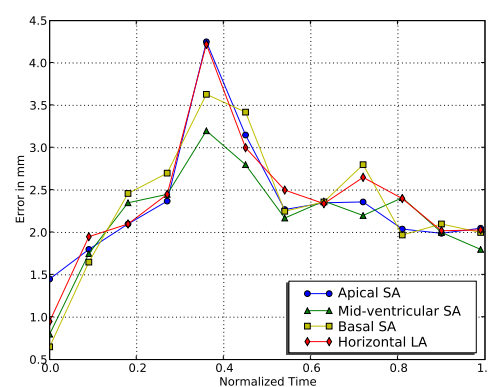

(b) Estimate with $50 \%$

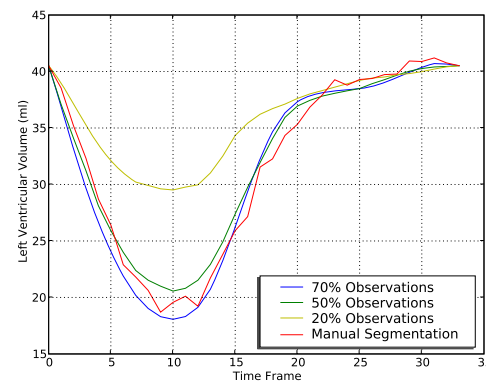

(d) LV volumes

Fig. 1. Comparison of the RMS errors over time for the motion estimation algorithm using partial observations. Motion estimation was performed using (a)70\%, (b) $50 \%$ and (c) $20 \%$ partial observations, and the errors are plotted as a function of time for each of the partial observation cases. (d) Left ventricular volume of a selected subject, segmented automatically and by manually over all frames in a cardiac cycle. 


\section{Conclusions}

We presented a method for cardiac motion reconstruction. We integrate taggedMR images and a biomechanical model that accounts for inhomogeneous tissue properties, fiber information, and active forces. We presented an inversion algorithm. Using only a total of 320 parameters we were able to reconstruct the $4 \mathrm{D}$ cardiac motion quite accurately.

The limitations of our current implementation (but not the method) is the assumptions of linear geometric and material response and the potential bias due to template-based fibers that does not account for anatomical variability, that is still requires some preprocessing of the initial frame to assign material properties and fiber orientation, that assumes zero residual stresses and initial conditions, and that it does not include an electrophysiology model.

Our on-going work includes transition to an intensity-based image-registration inversion (in which case we need to solve a nonlinear inversion) and its clinical validation by reconstructing motions of normal and abnormal populations and conducting statistical analysis. Among the many open problems are the level of required model complexity for clinically relevant motion reconstructions, the bias of the fibers, the sensitivity to the values of the material properties, and the sensitivity to the image similarity functional.

\section{References}

1. Castillo, E., Lima, J.A.C., Bluemke, D.A.: Regional myocardial function: Advances in MR imaging and analysis. Radiographics 23, S127-S140 (2003)

2. Shah, D.J., Judd, R.M., Kim, R.J.: Technology insight: MRI of the myocardium. Nature Clinical Practice Cardiovascular Medicine 2(11), 597-605 (2005)

3. O’Donnell, T., Funka-Lea, G., Tek, H., Jolly, M.P., Rasch, M., Setser, R.: Comprehensive cardiovascular image analysis using MR and CT at Siemens Corporate Research. IJCV 70(2), 165-178 (2006)

4. Sampath, S., Prince, J.L.: Automatic 3D tracking of cardiac material markers using slice-following and harmonic-phase MRI. Magnetic Resonance Imaging 25(2), 197208 (2007)

5. Axel, L.: Biomechanical dynamics of the heart with MRI. Annual Review of Biomedical Engineering 4, 321-347 (2002)

6. Hu, Z., Metaxas, D., Axel, L.: In vivo strain and stress estimation of the heart left and right ventricles from MRI images. Medical Image Analysis 7(4), 435-444 (2003)

7. Papademetris, X., Sinusas, A.J., Dione, D.P., Constable, R.T., Duncan, J.S.: Estimation of 3-D left ventricular deformation from medical images using biomechanical models. IEEE Trans. on Medical Imaging 21(7), 786 (2002)

8. Sermesant, M., Moireau, P., Camara, O., Sainte-Marie, J., Andriantsimiavona, R., Cimrman, R., Hill, D., Chapelle, D., Razavi, R.: Cardiac function estimation from MRI using a heart model and data assimilation: Advances and difficulties. Medical Image Analysis 10(4), 642-656 (2006)

9. Moireau, P., Chapelle, D., Le Tallec, P.: Joint state and parameter estimation for distributed mechanical systems. Computer Methods in Applied Mechanics and Engineering 197, 659-677 (2008) 
10. Sundar, H., Sampath, R.S., Biros, G.: Bottom-up construction and 2:1 balance refinement of linear octrees in parallel. SIAM Journal on Scientific Computing 30(5), 2675-2708 (2008)

11. Sundar, H., Sampath, R.S., Adavani, S.S., Davatzikos, C., Biros, G.: Lowconstant parallel algorithms for finite element simulations using linear octrees. In: ACM/IEEE SCXY Conference Series (2007)

12. Zitová, B., Flusser, J.: Image registration methods: a survey. Image Vision Comput. 21(11), 977-1000 (2003)

13. Gurtin, M.E.: An Introduction to Continuum Mechanics. Academic Press, London (1981)

14. Balay, S., Buschelman, K., Gropp, W.D., Kaushik, D., Knepley, M.G., McInnes, L.C., Smith, B.F., Zhang, H.: PETSc home page (2001), http://www.mcs.anl.gov/petsc

15. Sachse, F.B.: Computational Cardiology. LNCS, vol. 2966. Springer, Heidelberg (2004) 